\title{
Sclerosing encapsulating peritonitis after living donor liver transplantation: a case report
}

\author{
Jeong-lk Park' ${ }^{1}$ Bo-Hyun Jung ${ }^{2}$, Yong Kyu Chung ${ }^{2}$, Won Beom Jung ${ }^{3}$ \\ 'Department of Surgery-Hepatobiliary, Ulsan University Hospital, Ulsan, Korea \\ ${ }^{2}$ Department of Surgery-Hepatobiliary, Inje University Haeundae Paik Hospital, Busan, Korea \\ ${ }^{3}$ Department of Surgery, Inje University Haeundae Paik Hospital, Busan, Korea
}

Background: Sclerosing encapsulating peritonitis (SEP), or abdominal cocoon syndrome is a rare condition of unknown etiology in which intestinal obstruction results from encasement of variable lengths of bowel by a dense fibrocollagenous membrane. It is difficult to diagnose, and the prognosis is poor.

Case report: We report a case of SEP after living donor liver transplantation (LDLT) that was required surgical treatment. A 57-year-old male, that had undergone LDLT for alcoholic liver cirrhosis 15 months earlier, was admitted with intermittent abdominal pain and nausea. Computed tomography scan showed that large amount of ascites with peritoneal thickening and encasing small bowel loops. Symptoms have improved in conservative management. However, he was readmitted a week after discharge because the symptoms were occurred again. The patient underwent laparotomy 6 days after hospitalization. The surgical findings showed ascites, and encasement of whole bowels by a dense fibrocollagenous membrane. Adhesiolysis and surgical removal of the membrane were performed successfully. There was no complication during postoperative period, and he is healthy until now.

Conclusions: SEP occurs as consequence of persistent low-grade peritonitis and may complicated the postoperative course after LT. Long-term outcome is satisfactory after surgical removal of membrane, but perioperative mortality rates are significant.

Corresponding author: Jeong-Ik Park

E-mail: jipark@uuh.ulsan.kr

() The Korean Society for Transplantation

This is an Open Access article distributed under the terms of the Creative Commons Attribution Non-Commercial License (http://creativecommons.org/licenses/by-nc/4.0/) which permits unrestricted non-commercial use, distribution, and reproduction in any medium, provided the original work is properly cited. 